\title{
The Effect of High-Intensity Laser Therapy on Trapezius Focal Myositis: A Rare Case Report
}

\author{
Maryam Ghanbarnasab ${ }^{1}$, Parisa Nejati ${ }^{1 *}$, Reza Moeinoddin ${ }^{2}$, Lida Nejati ${ }^{3}$ \\ ${ }^{1}$ Sports Medicine Department, Rasool Akram Hospital, Iran University of Medical Sciences, Tehran, Iran \\ ${ }^{2}$ Dr. Moeinoddin Radiology and Sonography Clinic, Qazvin, Iran \\ ${ }^{3}$ Islamic Azad University, Science and Research Branch, Tehran, Iran
}

\author{
*Correspondence to \\ Parisa Nejati, \\ Department of Sports Medicine, Rasoo \\ Akram hospital, Niyayesh Street, \\ Sattarkhan street, Tehran, Iran \\ Tel: 0982164352446, 09125955643; \\ Fax: 0982166509108 \\ Email: parisanejati2@gmail.com
}

Published online October 1, 2019

\begin{abstract}
Focal myositis (FM) is a rare inflammatory myopathy characterized by a painful swelling of a skeletal muscle. The lower limb is the most common site for FM, but it has also been reported in the abdomen, forearm, neck, and tongue musculature. Some conservative treatments such as non-steroidal anti-inflammatory drugs, physiotherapy, and steroids can relieve muscle pain and its minor complications. This report describes a patient who had shoulder pain and was diagnosed with FM in the left trapezius muscle with an excellent response to high-intensity laser therapy.

Keywords: Focal myositis; High-intensity laser; Trapezius.
\end{abstract}

\section{Introduction}

Focal myositis (FM) is a rare, benign myopathy characterized by a focal inflammatory pseudo-tumor of the skeletal muscle, which was initially reported in 1977 by Heffner et al. ${ }^{1}$ It is uncertain whether FM is a unique clinical entity or a rare form of polymyositis. ${ }^{2}$ It presents itself as a painful swelling of a muscle that enlarges rapidly and simulates a soft tissue neoplasm. ${ }^{3}$ The leg is the most common site of FM, but it has also been reported in the abdomen, forearm, neck, and tongue musculature. ${ }^{3}$ Indeed, FM is recognized as a generalized inflammatory muscle disease, which may initially present itself focally, involving one limb at its onset. For example, Lederman described 3 patients with weakness of a single-limb myositis which progressed to generalized polymyositis. ${ }^{4}$ Barwick and Walton also described unilateral quadriceps weakness progressed to diffuse weakness. ${ }^{5}$

The laboratory findings of FM, including the erythrocyte sedimentation rate (ESR) and serum creatine kinase (CK) are usually normal. Computerized tomography (CT) reveals diffuse homogenous enlargement and fatty infiltration of the affected muscles. ${ }^{6}$ Magnetic resonance imaging (MRI) is one of the most useful diagnostic methods that show the abnormal signal intensity of the involved muscles. ${ }^{7}$ In some cases of FM, evidence of denervation has been reported in electromyography (EMG). ${ }^{2}$

We describe a case of FM in a rare place, namely the upper trapezius muscle, which was treated with a highpower laser with an excellent response.

\section{Case Report}

A 55-year-old woman was referred to the sports medicine clinic with severe shoulder pain. The pain had started in the left supraclavicular region spreading to the neck, ear, and occiput about 6 weeks before the treatment. It had been felt during the day and night and had caused her to wake up at times. She had been unable to sleep on the shoulder and sometimes even the pain had intensified by relying on the chair. The intensity of her pain was to the extent that she had been unable to perform her daily activities effectively. The score of the pain was 9 on the visual analog scale (VAS). There was not any history of trauma or special disease. However, she had had a history of falling about 6 years before the treatment, leading to a clavicle fracture which had been treated with an arm sling. Such systemic symptoms as general weakness, fever, and weight loss were not mentioned. In drug history, she had taken Aspirin $80 \mathrm{mg}$ daily for 10 years.

Physical examination revealed prominence area in the left supraclavicular region without evidence of redness or erythema. The left sternoclavicular joint was more prominent compared to the other side that seems compatible with the past clavicle fracture, but there was not any history of trauma in the area of pain. There was severe tenderness in the upper trapezius muscle, 
especially around its insertion into the clavicle and also in the left acromioclavicular joint. No nodule or mass was palpated. All ranges of shoulder active or passive motion were normal and pain free; however, the arm abduction and forward flexion were a little painful. The special tests of the glenohumeral joint, such as Job, Lift-off, BellyPress, Neer, Hawkins, and O'Brian tests were normal. The patient felt pain through the cross arm adduction test, the head rotation to the right and the length-tension test of the left upper trapezius muscle. The strength of the left trapezius muscle was not assessed due to the pain but on the right side, it was $5 / 5$ by the muscle manual test.

Regarding the symptoms and the findings of physical examination, a shoulder and cervical MRI and also some laboratory tests were requested. In the cervical MRI, an intervertebral disc protrusion was seen at the level of C5-6 without compressive effects on the nerve root, which was not compatible with the patient's symptoms.

There was a poorly defined, high-signal area in the attachment of trapezius muscle to the clavicle at the coronal and sagittal T2 sequences and also in the proton density fat suppress sequences. There was also an obvious isointense area in the T1 sequence of the shoulder MRI too (Figures 1 and 2).

The findings from the laboratory tests of collagen vascular diseases were normal. They included ANA (anti-nuclear antibody), anti double-stranded DNA, anti JO1, RF (rheumatoid factor), ESR, and CRP (C-reactive protein). Other tests such as CBC (complete blood count), LDH (lactate dehydrogenase), ALP (alkaline phosphatase), CPK (creatine phosphokinase), ALT (alanine aminotransferase), AST (aspartate aminotransferase), BUN (blood urea nitrogen), Creatine and U/A (urinary analysis) were also normal. Consequently, collagen vascular disease, rhabdomyolysis or other systemic diseases were considered fewer. In order to recognize the exact cause of the symptoms and signs, a muscle biopsy was advocated, but the patient rejected the offer. We prescribed $100 \mathrm{mg}$ diclofenac daily for 7 days, but unfortunately, the pain did not subside. As the next step, after 2 weeks, we decided to inject $40 \mathrm{mg}$ DepoMedrol (methylprednisolone acetate) plus $1 \mathrm{cc}$ lidocaine $2 \%$ in the maximum point of tenderness in the trapezius muscle. The pain and swelling were eliminated after 3 days slightly (the VAS declined to $8 / 10$ ). The patient was observed closely after injection. Given the weak response to oral Diclofenac and the injected Depo-Medrol, laser therapy started after 4 weeks. A high-intensity laser (ND-YAG, $1064 \mathrm{~nm}$ Physiomed, Belgium) was used through 8 sessions, every other day, after obtaining the informed consent. The pain was significantly reduced after 3-4 sessions (the VAS declined to 3/10). At the end of the eighth session, there was a dramatic decrease in the VAS score (1/10), and finally, the pain completely disappeared after 1 week. After three months, the patient was completely painless. The second shoulder MRI (with gadolinium contrast) was requested. The lesion was smaller than before (Figures 3 and 4 ). After 6 months, she had no pain or tenderness in that area and was able to perform all daily tasks without any pain.
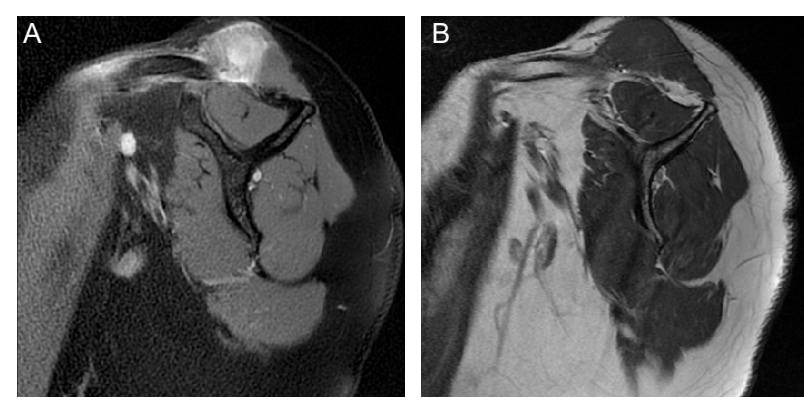

Figure 1. Sagittal view of the shoulder MRI; (A) PD, (B) T1.
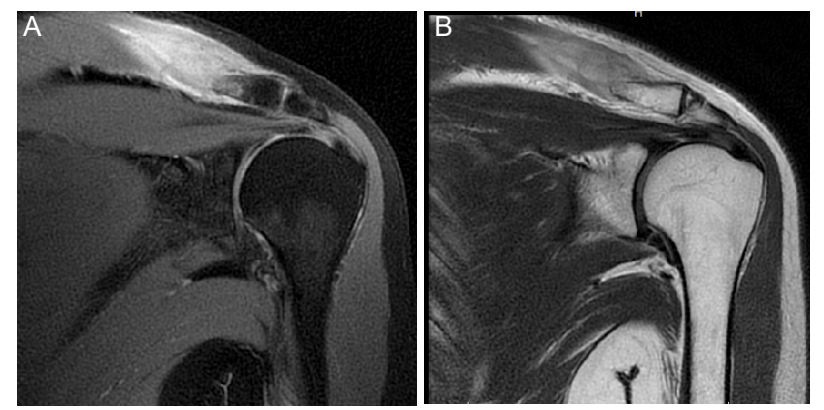

Figure 2. Coronal View of the Shoulder MRI; (A) PD, FS; (B) T2.
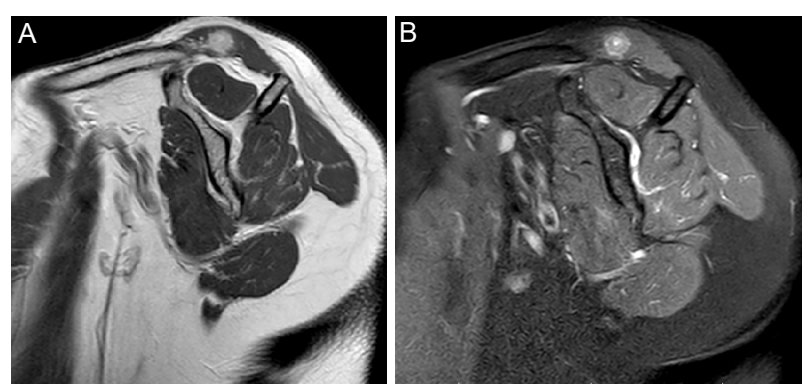

Figure 3. Sagittal view of the shoulder MRI; (A) T1, (B) PD.
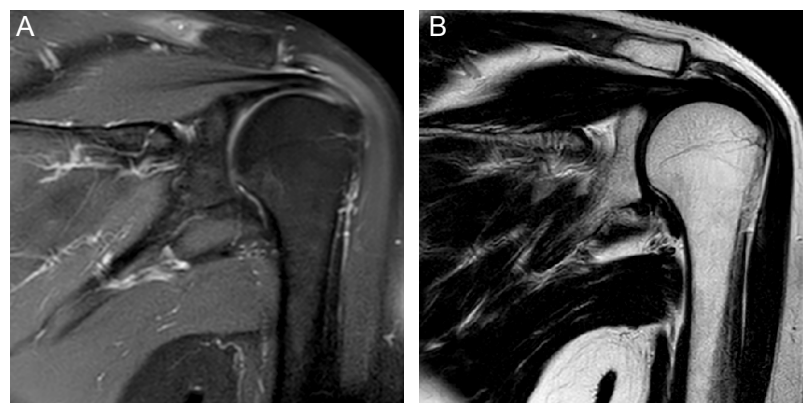

Figure 4. Coronal View of the Shoulder MRI; (A) PD (B) T1. 


\section{Discussion}

The case of this study was FM of a trapezius muscle with an excellent response to high-intensity laser therapy (HILT). Two cases of myositis in the trapezius muscle have also been reported, which responded very well to steroid therapy. One of them was isolated myositis that led to muscle atrophy and drop head syndrome and was recognized by clinical manifestations and histopathologic features. ${ }^{8}$ The other one was a case of myositis in the left and right trapezius muscles with eye involvement. ${ }^{9}$ Therefore, to the best of our knowledge, this study gains significance by presenting a rare case report of isolated FM in the left upper trapezius muscle with a wonderful response to a high-intensity laser.

The laser as a new modality has been used in the treatment of a variety of acute and chronic musculoskeletal injuries, degenerative conditions, and wound healing. ${ }^{10}$ Ramos et al studied the effect of laser photobiomodulation on muscle injury in rats. They used male Wistar rats (200 g) and performed tibialis muscle elongation by passive stretching of the anterior tibial muscle attached to a weight corresponding to $150 \%$ of the body mass of the animal. The stretching continued for 20 minutes and with a 3 minutes' rest the second traction was repeated for 20 minutes again. Cytokines, tumor necrosis factor- $\alpha$ (TNF- $\alpha$ ), interleukin-1 $\beta$ (IL-1 $\beta$ ), IL-6, and IL-10, edema, and C-reactive protein (CRP) levels were determined in their tibialis anterior muscles. Finally, they concluded that the laser reduced skeletal muscle edema as well as cytokines, and CRP then led to a significant reduction in inflammatory markers. ${ }^{11}$

Through a study guided by Carvalho et al, 15 male rats were distributed into three groups: control group (C), induced myositis without treatment (MI), and treated induced myositis (MT), for seven days. The induction of acute myositis was done with $1 \%$ acetic acid by the intramuscular route. The rats in the MT group had daily treatment through the GaAs (gallium arsenide) laser, with $904 \mathrm{~nm}$ length and $45 \mathrm{~mW}$ peak power with $3 \mathrm{~J} /$ $\mathrm{cm}^{2}$, applied for 5 minutes to the animals' right posterior limb. In the MT group, there was a statistically significant decrease in the number of inflammatory cells $(P<0.05)$ compared to the MI group. In addition, the MT group showed an increase in the fibroblastic proliferation compared to $\mathrm{C}$ and $\mathrm{MI}$ groups $(P<0.01)$. Also, significant edema regression $(P=0.040)$ in the MT group achieved similar results in rats. ${ }^{12}$ Moreover, the positive effects of laser therapy have been seen in a few cases of dermatomyositis. ${ }^{13}$

HILT which includes higher intensity laser radiation is a new, painless, and powerful modality and it has shown significant results concerning pain reduction in the osteoarthritis and other musculoskeletal disorders. ${ }^{14}$ However, the evidence of the effectiveness of the highintensity laser in musculoskeletal pain is limited because the number of related studies is low. Alayat et al assessed the effect of the ND YAG laser on knee osteoarthritis. They concluded the pulsed Nd:YAG laser combined with glucosamine/chondroitin sulfate (GCS) and exercise were more effective than GCS plus exercise (GCS + EX) and exercise alone in the treatment of knee osteoarthritis. ${ }^{15}$

HILT has satisfactory effects on reducing pain. HILT through photo-chemical, photo-thermal, and photomechanical actions has many therapeutic benefits including antalgic, anti-edema, and bio-stimulating effects. ${ }^{16,17}$ Another advantage of HILT, especially the neodymium-doped yttrium aluminum garnet (ND YAG) laser, is its greater power and depth of penetration in deep tissues. $^{18}$

Therefore, our case showed that HILT was an effective method in the pain relief of myositis. Its effectiveness can be due to its analgesic and anti-inflammatory effects. Nevertheless, more extensive studies are needed to prove the efficacy of HILT and compare the low-level laser with HILT.

\section{Ethical Considerations}

An informed consent statement was taken from the patient for publication of this report.

\section{Conflict of Interests}

The authors declare no conflict of interest.

\section{References}

1. Heffner RR Jr, Armbrustmacher VW, Earle KM. Focal myositis. Cancer. 1977;40(1):301-6.

2. Wang J, Jiao J, Zhao G, Shi J, Xia J. Case report: A rare case of focal myositis presenting as Sartorius muscle contracture: A case report and review of literature. Medicine (Baltimore). 2018;97(20):e10766. doi: 10.1097/MD.0000000000010766.

3. Sekiguchi K, Kanda F, Oishi K, Hamaguchi H, Nakazawa $\mathrm{K}$, Maeda N, et al. HLA typing in focal myositis. J Neurol Sci. 2004;227(1):21-5. doi:10.1016/j.jns.2004.07.020

4. Lederman RJ, Salanga VD, Wilbourn A J, Hanson MR, Dudley AW Jr. Focal inflammatory myopathy. Muscle Nerve. 1984:7(2):142-6. doi:10.1002/mus.880070210

5. Barwick DD, Walton JN. Polymyositis. Am J Med. 1963;35,(5):646-60.

6. Smith AG, Urbanits S, Blaivas M, Grisold W, Russell JW. Clinical and pathologic features of focal myositis. Muscle Nerve. 2000;23(10):1569-75.

7. Colding-Jørgensen E, Laursen H, Lauritzen M. Focal myositis of the thigh: report of two cases. Acta Neurol Scand. 1993;88(4):289-92.doi: 10.1111/j.1600-0404.1993. tb04238.x

8. Kastrup A, Gdynia HJ, Nägele T, Riecker A.Dropped-head syndrome due to steroid responsive focal myositis: a case report and review of the literature. J Neurol Sci. 2008;267(12):162-5. doi: 10.1016/j.jns.2007.09.042

9. Guerrero M, Manrique A, Del Rio S, Ruiz S, Haro E, Díaz L, et al. Sign of the school knapsack: myositis of the trapezius muscles caused by overload. Clin Nucl Med. 2000;25(4):2967. doi:10.1097/00003072-200004000-00016

10. Dawood MS, Al-Salihi AR, Qasim AW. Laser therapy of 
muscle injuries. Lasers Med Sci. 2013 ;28(3):735-42. doi: 10.1007/s10103-012-1131-2.

11. Ramos L, Marcos RL, Torres-Silva R, Pallota RC, Magacho T, Mafra FFP, et al. Characterization of Skeletal Muscle Strain Lesion Induced by Stretching in Rats: Effects of Laser Photobiomodulation. Photomed Laser Surg. 2018;36(9):460-467. doi: 10.1089/pho.2018.4473.

12. Carvalho AF, Sousa PF, Feitosa MC, Coelho NP, Barros EM, Feitosa VC, et al. The low-level laser on acute myositis in rats. Acta Cir Bras. 2015;30(12):806-11. doi:10.1590/ S0102-865020150120000003

13. Brauer JA, Gordon Spratt EA, Geronemus RG. Laser therapy in the treatment of connective tissue diseases: a review. Dermatol Surg. 2014;40(1):1-13. doi: 10.1111/ dsu.12339.

14. Nazari A, Moezy A, Nejati P, Mazaherinezhad A. Efficacy of high-intensity laser therapy in comparison with conventional physiotherapy and exercise therapy on pain and function of patients with knee osteoarthritis: a randomized controlled trial with 12-week follow up. Lasers Med Sci. 2019;34(3):505-516. doi: 10.1007/s10103-0182624-4.

15. Alayat MS, Aly TH, Elsayed AE, Fadil AS. Efficacy of pulsed Nd:YAG laser in the treatment of patients with knee osteoarthritis: a randomized controlled trial. Lasers Med Sci. 2017;32(3):503-511. doi: 10.1007/s10103-017-2141-x.

16. Stiglić-Rogoznica N1, Stamenković D, Frlan-Vrgoc L, Avancini-Dobrović V, Vrbanić TS. Analgesic effect of high-intensity laser therapy in knee osteoarthritis. Coll Antropol. 2011;35 Suppl 2:183-5.

17. Kheshie AR, Alayat MS, Ali MM. High-intensity versus low-level laser therapy in the treatment of patients with knee osteoarthritis: a randomized controlled trial. Lasers Med Sci. 2014;29(4):1371-6. doi: 10.1007/s10103-0141529-0.

18. Fan ZW, Qiu JS, Kang ZJ, Chen YZ, Ge WQ, Tang XX. High beam quality $5 \mathrm{~J}, 200 \mathrm{~Hz} \mathrm{Nd}$ :YAG laser system. Light Sci Appl 2017;6(3): e17004. 